\title{
Decision-to-delivery interval of emergency cesarean section in Uganda: a retrospective cohort study
}

\author{
Noemi J. Hughes ${ }^{1}$, Imelda Namagembe ${ }^{2}$, Annettee Nakimuli², Musa Sekikubo², Ashley Moffett ${ }^{3}$,
} Charlotte J. Patient ${ }^{4}$ and Catherine E. Aiken ${ }^{1,4,5^{*}}$ (1)

\begin{abstract}
Background: In many low and medium human development index countries, the rate of maternal and neonatal morbidity and mortality is high. One factor which may influence this is the decision-to-delivery interval of emergency cesarean section. We aimed to investigate the maternal risk factors, indications and decision-to-delivery interval of emergency cesarean section in a large, under-resourced obstetric setting in Uganda.

Methods: Records of 344 singleton pregnancies delivered at $\geq 24$ weeks throughout June 2017 at Mulago National Referral Hospital were analysed using Cox proportional hazards models and multivariate logistic regression models.

Results: An emergency cesarean section was performed every $104 \mathrm{~min}$ and the median decision-to-delivery interval was $5.5 \mathrm{~h}$. Longer interval was associated with preeclampsia and premature rupture of membranes/oligohydramnios. Fetal distress was associated with a shorter interval $(p<0.001)$. There was no association between decision-to-delivery interval and adverse perinatal outcomes $(p>0.05)$. Mothers waited on average $6 \mathrm{~h}$ longer for deliveries between 00:0008:00 compared to those between 12:00-20:00 $(p<0.01)$. The risk of perinatal death was higher in neonates where the decision to deliver was made between 20:00-02:00 compared to 08:00-12:00 $(p<0.01)$.

Conclusion: In this setting, the average decision-to-delivery interval is longer than targets adopted in high development index countries. Decision-to-delivery interval varies diurnally, with decisions and deliveries made at night carrying a higher risk of adverse perinatal outcomes. This suggests a need for targeting the improvement of service provision overnight.
\end{abstract}

Keywords: Africa, Cesarean, Decision, Emergency, Obstetrics, Perinatal, Uganda

\footnotetext{
* Correspondence: cema2@cam.ac.uk

'School of Clinical Medicine, University of Cambridge, NIHR Cambridge Comprehensive Biomedical Research Centre, Cambridge CB2 OSW, UK

${ }^{4}$ Department of Obstetrics and Gynecology, Box 223, The Rosie Hospital, Cambridge CB2 OSW, UK

Full list of author information is available at the end of the article
}

C C The Author(s). 2020 Open Access This article is licensed under a Creative Commons Attribution 4.0 International License, which permits use, sharing, adaptation, distribution and reproduction in any medium or format, as long as you give appropriate credit to the original author(s) and the source, provide a link to the Creative Commons licence, and indicate if changes were made. The images or other third party material in this article are included in the article's Creative Commons licence, unless indicated otherwise in a credit line to the material. If material is not included in the article's Creative Commons licence and your intended use is not permitted by statutory regulation or exceeds the permitted use, you will need to obtain permission directly from the copyright holder. To view a copy of this licence, visit http://creativecommons.org/licenses/by/4.0/. The Creative Commons Public Domain Dedication waiver (http://creativecommons.org/publicdomain/zero/1.0/) applies to the data made available in this article, unless otherwise stated in a credit line to the data. 


\section{Summary box}

\section{A. What is already known?}

- In low human development index settings, emergency cesarean section is often associated with high rates of maternal and neonatal morbidity and mortality.

- Decision-to-delivery interval of emergency cesarean section is a modifiable factor which can influence perinatal outcomes.

\section{B. What are the new findings?}

- Average decision-to-delivery intervals are long in this setting compared to international guidelines.

- There is no direct association between decision-todelivery interval and risk of adverse outcomes.

- There is diurnal variation in both decision-todelivery intervals and adverse outcomes, with both increasing overnight.

\section{What do the new findings imply?}

- Reducing the diurnal variation in decision-todelivery interval could improve perinatal outcomes of emergency caesarean section.

- We suggest this could be achieved by altering patterns of service provision to better support deliveries and decision-making overnight.

\section{Background}

In many low and medium human development index (LM-HDI [1]) countries, the rate of maternal and neonatal morbidity and mortality is high $[2,3]$. Preventing adverse perinatal outcomes is often critically time-dependent, however, demand can exceed capacity for prompt intervention in these settings wall]. Despite global initiatives for improvement, there remains considerable complexity in increasing obstetrics resource availability in LM-HDI settings $[4,5]$. We considered whether improving patterns of service provision might provide an alternative strategy to reduce adverse perinatal outcomes in LM-HDI settings. Studies have shown that risk of adverse perinatal outcomes is associated with service provision factors such as obstetric staff working patterns [6,7]. This has only, however, been studied extensively in well-resourced countries and so there is a need to investigate the modifiable patterns of service provision in low and medium human development index obstetric settings.
One aspect of service provision with the potential to influence perinatal outcome is the decision-to-delivery interval of emergency cesarean section [8]. National guidelines in the USA and UK suggest a target of $30 \mathrm{~min}$ after the decision to deliver by emergency cesarean section is established $[9,10]$. Such guidelines, however, are not wellevidenced [11] and may not be feasible even in wellresourced obstetric settings [12]. Furthermore, whilst globally it is generally accepted that the decision-todelivery interval should be kept to the minimum time achievable [13], there are currently no context-appropriate targets intended to minimise adverse outcomes in low and medium human development index countries.

Mulago National Referral Hospital is a governmentrun facility with over 2700 beds [14] in Kampala, Uganda. The birth rate has been reported to exceed 39, 000 per annum [15]. Approximately 22\% of all deliveries in the study centre are by caesarean section, of which $85 \%$ are by emergency cesarean section [16]. This high volume combined with the socio-economic challenges [15], poor baseline health status [17, 18], and lack of antenatal care [19] experienced by mothers presenting to the hospital, has resulted in high rates of maternal and neonatal morbidity and mortality [20,21]. Studies from this setting have shown that obstetric service provision is not uniform during a 24-h shift cycle [22]. We therefore aimed to investigate whether the modifiable factor of decision-to-delivery interval is associated with adverse perinatal outcomes.

\section{Methods}

Mothers who delivered a singleton pregnancy, at viable gestational age ( $\geq 24$ completed weeks), by emergency cesarean section during the period of June 2017 were included in the study. We analysed their full medical records for the entire delivery episode from admission to discharge. Data was collected contemporaneously in fully anonymised form and the data used for analysis is summarised in Table 1. Mothers who died following emergency cesarean section were excluded from the study $(n \leq 3)$, due to possession of their records by other investigatory authorities. Cases of intrauterine death in which the fetus was thought to have demised prior to presentation at the hospital were also excluded, as this outcome could not have been affected by decision-to-delivery interval. The diagnosis of fetal demise prior to presentation was made according to the contemporaneous judgement of the attending clinician. The diagnosis was based primarily on the macerated appearance of the fetus after delivery, but also took into account factors such as whether the fetal heart was ever auscultated and the maternal history. A typical shift at the study centre is staffed by an attending obstetrician, 3-4 resident obstetricians, and one intern. For all included deliveries, the 
Table 1 Summary of data extracted from contemporaneous medical notes

\begin{tabular}{|c|c|}
\hline Data & Details \\
\hline Maternal age & Self-reported by mother or referring clinician. \\
\hline Gestational age & $\begin{array}{l}\text { Calculated from the date of last menstrual period stated by mother or symphysial-fundal height. Routine } \\
\text { first trimester US is not available in this context. }\end{array}$ \\
\hline Previous cesarean section & Evidenced by an existing abdominal scar, with maternal report. \\
\hline Comorbidities (composite factor) & $\begin{array}{l}\text { One or more of HIV, active malaria and sickle-cell crisis as stated by mother or diagnosed by attending } \\
\text { clinician. }\end{array}$ \\
\hline Pre-eclampsia & $\begin{array}{l}\text { Diagnosed according to modified ACOG guidelines [16] - blood testing is not routinely available for } \\
\text { investigating suspected pre-eclampsia, therefore the criteria based on biochemical results were not } \\
\text { applied. }\end{array}$ \\
\hline Antepartum haemorrhage & Any fresh vaginal blood loss reported by the mother prior to delivery \\
\hline $\begin{array}{l}\text { Premature rupture of membranes / } \\
\text { oligohydramnios }\end{array}$ & $\begin{array}{l}\text { Premature rupture of membranes based on maternal history, oligohydramnios was diagnosed by } \\
\text { clinicians on the basis of clinical examination }+/- \text { ultrasound scan }\end{array}$ \\
\hline Uterine rupture & Based on clinical suspicion at the time of decision-making \\
\hline Obstructed labour & $\begin{array}{l}\text { Diagnosed by the decision-making clinician based on examination (e.g. excessive fetal caput, haematuria) } \\
\text { or history (e.g. length of time in labor) }\end{array}$ \\
\hline Fetal distress & $\begin{array}{l}\text { Diagnosed by the decision-making clinician based on clinical suspicion e.g. meconium stained liquor or } \\
\text { decelerations on intermittent auscultation. Continuous fetal monitoring, and fetal blood sampling were } \\
\text { not available }\end{array}$ \\
\hline Malpresentation & Diagnosed by the delivering clinician \\
\hline Cord prolapse & Diagnosed by the delivering clinician \\
\hline Decision & $\begin{array}{l}\text { Date and time at which the decision to deliver by emergency cesarean section was recorded in the } \\
\text { contemporaneous medical notes. }\end{array}$ \\
\hline Delivery & Date and time at which the neonate was delivered according to the operation note \\
\hline Decision-to-delivery interval & Calculated to the nearest minute \\
\hline Adverse maternal outcome (composite) & $\begin{array}{l}\text { One or more: confirmed uterine rupture at delivery, severe postpartum haemorrhage ( } \geq 1 \mathrm{~L} \text { blood), } \\
\text { emergency hysterectomy, admission to the High-Dependency Unit or obstetric palsy }\end{array}$ \\
\hline Neonatal APGAR scores & Recorded at 1 and 5 min \\
\hline Stillbirth & Viable baby born with no signs of life that was believed to have been alive at admission to hospital \\
\hline Neonatal death & Live birth at viable gestational age, followed by death prior to hospital discharge \\
\hline Perinatal death (composite) & All stillbirths and neonatal deaths (defined as above) \\
\hline Adverse neonatal outcome (composite) & One or more of birth asphyxia, resuscitation, birth trauma and respiratory distress \\
\hline Gravidity & Self-reported number of previous pregnancies \\
\hline Parity & Self-reported number of previous deliveries $\geq 24$ weeks \\
\hline Birth weight & Recorded to the nearest $100 \mathrm{~g}$ \\
\hline Neonatal sex & As recorded in contemporaneous medical record \\
\hline
\end{tabular}

decision was made by an attending doctor to deliver on an emergency basis by caesarean section. Neonatalogy services are available at the study centre.

Using binary logistic regression, we compared the characteristics of deliveries occurring during the day to those during the night and those during weekends to weekdays. Using univariate and multivariate Cox proportional hazard models, we assessed the relationship between both maternal risk factors and indications for each emergency cesarean section and the decision-to-delivery interval. These models accounted for time-at-risk of adverse outcomes. Using binomial regression models, we assessed the relationship between adverse perinatal outcomes and the decision-to-delivery interval. Using generalised additive models (in which all events were considered equivalent), we assessed the relationship between (i) time of decision, (ii) time of delivery, and (iii) decision-to-delivery interval, and adverse outcomes. The generalised additive models incorporate a nonlinear term for event time on the risk of each adverse outcome and this was estimated using cubic splines. All events included in the modelling were considered equivalent. Using non-parameter models avoids the requirement to make any assumptions about the nature of the relationship between the timing of an event and the risk of an adverse outcome. The risk of an event at any particular time, relative to the average population risk, can therefore be assessed from the graphical representations of the models presented in the figures. 
All multivariate models were adjusted for covariates selected on the basis of clinical relevance and using Akaike Information Criterion (AIC) to optimise model fit. Statistical significance of the nonlinear effect of time of delivery was assessed using a likelihood-ratio test. To summarise our findings, Kaplan-Meier curves were constructed to represent the decision-to-delivery interval of the entire population and of relevant sub-cohorts. Our findings were considered statistically significant at an alpha level of 0.05 . Power calculations were performed by Monte Carlo simulation. All analyses were conducted using the R statistical software package version 3.5.1.

\section{Results}

During the study period of June 2017, 412 mothers underwent an emergency cesarean section at Mulago Hospital. Of these, 396 delivered a singleton pregnancy at viable gestational age ( $\geq 24$ completed weeks) and 349 of these records had complete information regarding the decision-to-delivery interval. 5 observations were removed from the time-dependent analysis on the basis that their status as an emergency was reversed prior to delivery. In these 5 cases, the neonate was delivered more than 4 days after the initial decision time. A delivery by emergency cesarean section occurred on average every $104 \mathrm{~min}$ (median 13.7 per day) throughout the entire study period. The median decision-to-delivery interval was $5.5 \mathrm{~h}$, with interquartile range 3.3-10.7 and range $0.5-92.3 \mathrm{~h}$ (Fig. 1). 2\% (7/344) of neonates were delivered within $1 \mathrm{~h}$ of decision-making.

The average number of decisions made per hour varied significantly throughout the day (minimum: 0.03 decisions per hour 05:00-06:00, maximum: 1.1 decisions per hour between 12:00-13:00, $p<0.001$, Fig. 2a). The average number of deliveries also varied significantly (minimum

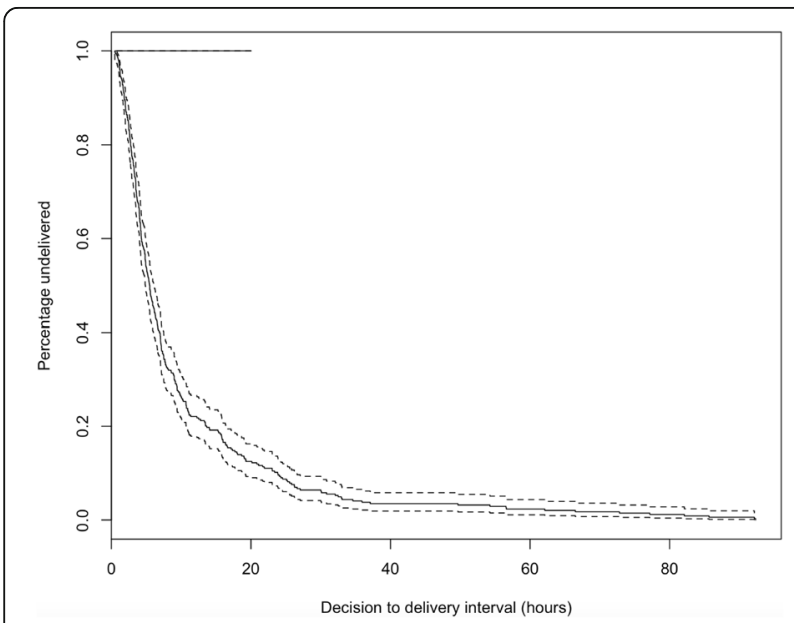

Fig. 1 Percentage of emergency cesarean section completed by time from decision-making. Median decision-to-delivery interval: $5.5 \mathrm{~h}$ (IQR 3.3-10.7 h). Mean decision-to-delivery interval: 10.2 h (S.D. \pm 13.9 h)
0.03 deliveries per hour 08:00-10:00, maximum 0.87 deliveries per hour between 19:00-20:00, $p<0.001$, Fig. 2b). The average length of decision-to-delivery interval also varied significantly throughout the day (minimum $3.3 \mathrm{~h}$ 16:00-17:00, maximum 9.4 h 01:00-02:00, $p<0.01$, Fig. 3). Specifically, mothers who delivered during the night (between 0:00 and 08:00) waited on average $2 \mathrm{~h}$ longer for their emergency cesarean section compared to the rest of the cohort. There was a 5.6-h difference in average length of interval between those delivering during the longest wait times (00:00 to 08.00) and shortest wait times (12,00 to 20.00) (Fig. 3). There was no significant difference in the interval on any particular day of the week or at the weekend.

None of the risk factors known prior to birth, including preeclampsia, were significantly associated with shorter decision-to-delivery intervals using univariate analysis (Table 2). Longer interval was associated only with preeclampsia $(p<0.05)$ and premature rupture of the membranes / oligohydramnios $(p<0.01)$ on univariate analysis. After adjustment for relevant covariates, both preeclampsia (HR 0.61; CI 0.38-0.97, $p<0.05$ ) and premature rupture of the membranes / oligohydramnios (HR 0.60; CI 0.37-0.96, $\mathrm{p}<0.05$ ) retained significance.

We examined whether individual indications for emergency cesarean section were associated with the decisionto-delivery interval (Table 3) with univariate analysis. Fetal distress was associated with a shorter interval $(p<0.05$, Fig. 4a) whilst preeclampsia was associated with a longer interval ( $p<0.05$, Fig. $4 b)$. After adjustment for relevant covariates in multivariate models, fetal distress (HR 1.63, CI $1.23-2.15 ; p<0.001$ ), previous cesarean section (HR 1.66, CI 1.24-2.21; $p<0.01$ ), malpresentation (HR 1.78, CI 1.17-2.69; $\mathrm{p}<0.01$ ), antepartum haemorrhage (HR 1.56, CI $1.00-2.43, P<0.05)$ and impending uterine rupture (HR 1.85, CI $1.24-2.78 ; p<0.05$ ) were all significantly associated with shorter interval.

None of the maternal or fetal adverse outcomes measured showed a linear relationship with the decision-todelivery interval (Table 4). There was, however, a significant association between the timing of a decision or delivery and adverse perinatal outcomes. This included the outcomes of both perinatal (Fig. 5a-b; $\boldsymbol{p}<0.05$ ) and neonatal (Fig. $5 \mathrm{c}-\mathrm{d} ; \boldsymbol{p}<\mathbf{0 . 0 5}$ ) death. The risk of both perinatal death and neonatal death varied by $>50 \%$ between the highest and lowest risk periods (Fig. 5a-d). The lowest risk decision period was in the morning between 08:00 and noon $(p<0.01)$, whereas the lowest risk delivery period was in the afternoon between noon and 20:00 $(p<0.05)$. The highest risk decisions and deliveries both occurred between 22:00 and 04:00. The risk of both perinatal death $(p<0.05)$ and neonatal death $(\mathrm{p}<0.05)$ showed significantly stronger association with the decision time than delivery time. 

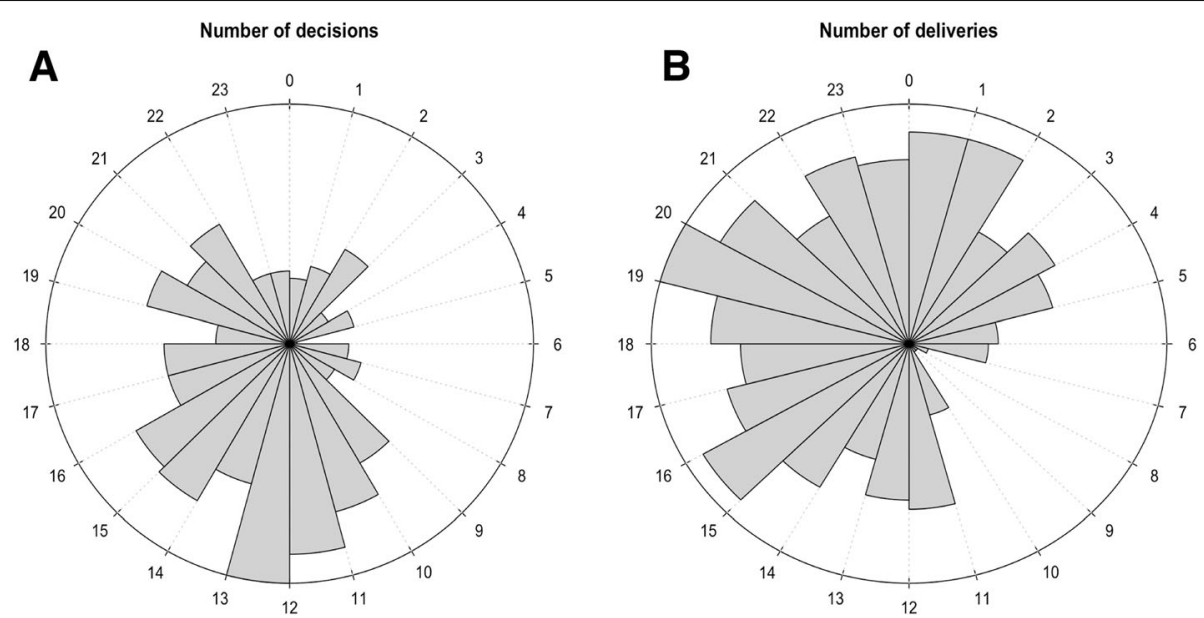

Fig. 2 Distribution of emergency cesarean section throughout the 24-h period. a) Number of decisions for emergency cesarean section by hour. There was significant variation in the average number of decisions per hour throughout the day $(p<0.001)$. $\mathbf{b})$ Number of deliveries by emergency cesarean section. There was significant variation in the average number of deliveries per hour throughout the day $(p<0.001)$

\section{Discussion}

In this obstetric setting, the average decision-to-delivery interval was $5.5 \mathrm{~h}$, with only $2 \%$ of babies delivered within an hour of decision-making. Women with certain indications were delivered more quickly once the decision for emergency cesarean section was made. Fetal distress, malpresentation, antepartum haemorrhage, and previous cesarean section (both with and without concern regarding impending uterine rupture) were prioritised over other indications. Whilst there was no association between the length of the decision-to-delivery interval and adverse perinatal outcomes, both the interval and risk of perinatal mortality showed significant diurnal variation. The time of decision-making was better correlated with the risk of adverse perinatal outcome that the time of delivery.

Accounting for the time taken to clean and restock the theatres between cases, we observed a remarkable continuous rate of emergency surgery in this LM-HDI setting. The average decision-to-delivery interval was significantly longer than targets adopted in well-resourced obstetric settings [10] as well as the average interval reported in other low resource contexts globally [13, 23]. However inconsistent

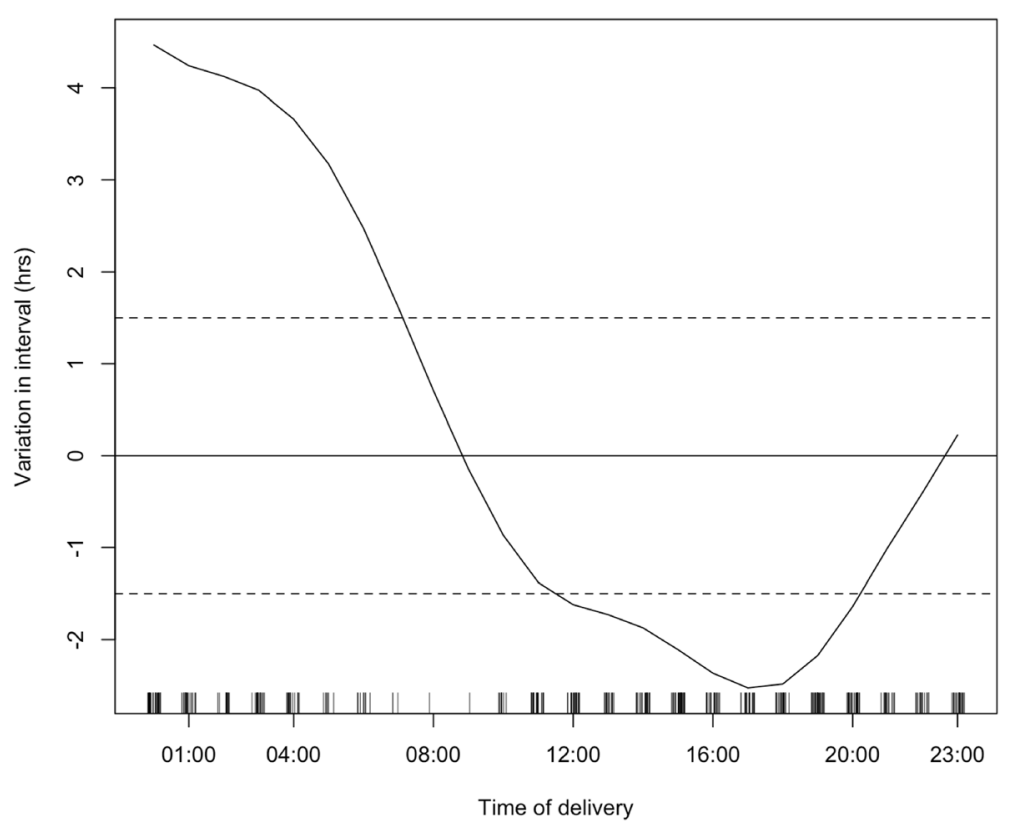

Fig. 3 Average decision-to-delivery interval by time of delivery. Solid line: median decision-to-delivery interval. Dashed lines: \pm standard errors, decision-to-delivery interval varies significantly over the 24 -h period; $p<0.01$ 
Table 2 Factors known prior to delivery

\begin{tabular}{|c|c|c|c|c|}
\hline Maternal factor & & Characteristic $(n=344)$ & Impact on decision-to-delivery interval & Significance \\
\hline Age & & $25.4 \pm 5.1$ & $0.99(0.97-1.02)$ & 0.59 \\
\hline Gestational age & & $37.57 \pm 2.0$ & $1.05(0.99-1.11)$ & 0.06 \\
\hline Parity & 0 & $130(37.8 \%)$ & Ref & \\
\hline & 1 & $81(23.5 \%)$ & $0.93(0.71-1.23)$ & 0.63 \\
\hline & 2 & $65(18.9 \%)$ & $0.97(0.71-1.31)$ & 0.83 \\
\hline & $\geq 3$ & $68(19.8 \%)$ & $1.02(0.75-1.34)$ & 0.91 \\
\hline Co-morbidities & No & $339(98.5 \%)$ & Ref & \\
\hline & Yes & $4(1.5 \%)$ & $1.25(0.52-3.03)$ & 0.62 \\
\hline Previous cesarean section & No & $196(57 \%)$ & Ref & \\
\hline & Yes & $148(43 \%)$ & $0.92(0.74-1.14)$ & 0.44 \\
\hline Previous poor neonatal outcome & No & $334(97.1 \%)$ & Ref & \\
\hline & Yes & $10(2.9 \%)$ & $1.17(0.61-2.21)$ & 0.64 \\
\hline Preeclampsia & No & $323(93.9 \%)$ & Ref & \\
\hline & Yes & $21(6.1 \%)$ & $0.62(0.39-0.98)$ & $0.04^{*}$ \\
\hline $\mathrm{APH}$ & No & $325(94.5 \%)$ & Ref & \\
\hline & Yes & $19(5.5 \%)$ & $1.29(0.81-2.05)$ & 0.28 \\
\hline PROM/ oligohydramnios & No & $322(93.6 \%)$ & Ref & \\
\hline & Yes & $22(6.4 \%)$ & $0.55(0.36-0.86)$ & $<0.01^{* *}$ \\
\hline
\end{tabular}

Numeric characteristics are shown as mean \pm standard deviation. Categorical characteristics are shown as $\mathrm{n}$ (\%). Impact on decision-to-delivery interval is represented by the hazard ratio and confidence intervals from a Cox proportional hazards model conditioned only on the characteristic of interest. Significance is the $p$ value derived from the same model. ${ }^{*} p<0.05,{ }^{* *} p<0.01$

categorisation of cases as 'emergencies' [24] and different obstetric populations complicate international comparisons.

There was significant diurnal variation in decision-todelivery interval and risk of perinatal death, which may reflect the fluctuating availability of senior clinicians during a 24-h period. As in many obstetric settings globally, the most experienced obstetricians at Mulago Hospital are available for ward rounds and decisionmaking during the day, however, not overnight. In line with this, the times of shortest interval occurred during normal working hours (12:00-20:00). The period with fewest decisions for emergency cesarean section were made (22:00-02:00) corresponded to the times of highest perinatal mortality, whilst the period when the rate of decision-making was increasing most rapidly (09:0013:00) was associated with the lowest risk.

Reducing the average decision-to-delivery interval by $5 \mathrm{~h}$ in keeping with the 30 -min targets set elsewhere $[9,10]$ is

Table 3 Indications for emergency cesarean section

\begin{tabular}{llll}
\hline Indication for emergency cesarean section & Number $(\%, n=344)$ & Average decision-to-delivery interval & Significance \\
\hline Previous cesarean section (no suspicion of rupture) & $87(25.3 \%)$ & $5.1(3.0-9.4)$ & 0.08 \\
Previous cesarean section (suspicion of rupture) & $35(10.2 \%)$ & $5.2(3.3-8.3)$ & 0.19 \\
Obstructed labour & $172(50.0 \%)$ & $5.5(3.5-10.7)$ & 0.70 \\
Fetal distress & $80(23.3 \%)$ & $4.9(3.3-7.7)$ & $<0.05^{*}$ \\
Malpresentation (54\% breech) & $26(7.6 \%)$ & $4.0(2.7-7.0)$ & 0.06 \\
Antepartum haemorrhage / placenta praevia / accreta & $23(6.7 \%)$ & $3.3(1.9-11.0)$ & 0.34 \\
Preclampsia & $21(6.1 \%)$ & $8.6(3.2-17.4)$ & $<0.05^{*}$ \\
Cord prolapse & $5(1.5 \%)$ & $6.3(4.9-6.6)$ & 0.65 \\
Other & $10(2.9 \%)$ & $7.3(6.0-27.8)$ & 0.07 \\
\hline
\end{tabular}

The number of mothers with each indication for emergency cesarean section along with the percentage of the analytic cohort is shown. More than one indication was present in many cases. The median decision-to-delivery interval and IQR range are shown for each indication. The impact of each indication on decision-todelivery interval is represented by the $p$-values from a Cox proportional hazards model conditioned only on the indication of interest. Significance is the $\mathrm{p}$ value derived from the same model. ${ }^{*} p<0.05$ 
A

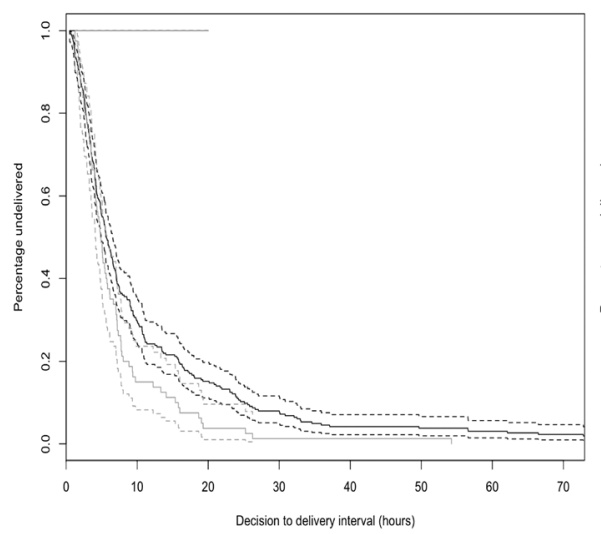

B

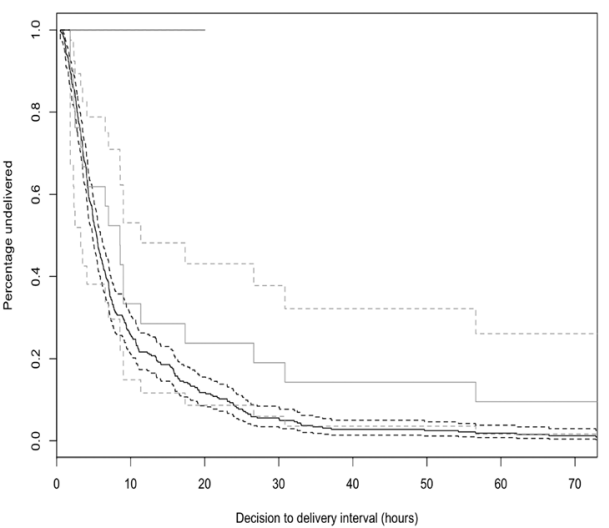

Fig. 4 decision-to-delivery interval by indication for emergency cesarean section. a) Solid grey line: deliveries where fetal distress was an indication, dashed grey lines: 95\% confidence intervals, solid black line: all deliveries without fetal distress as an indication, dashed black lines: $95 \%$ confidence intervals. $p<0.05$. b) Solid grey line: deliveries where preeclampsia was an indication for emergency cesarean section, dashed grey lines: $95 \%$ confidence intervals, solid black line: all deliveries without preeclampsia as an indication, dashed black lines: $95 \%$ confidence intervals. $p<0.05$

unlikely to be feasible in our already under-resourced study setting. Moreover, existing guidelines are not wellevidenced [11] with little direct evidence of benefit even in well-resourced obstetric setting. We did not find evidence of a direct relationship between longer interval and adverse perinatal outcomes, therefore it is unlikely to be of benefit to focus scarce resources towards dramatically reducing absolute time to delivery. Rather, our data support the idea that clear, timely, and well-supported clinical decisionmaking may have more influence on perinatal outcomes. Normal working hours also correspond to the highest availability of non-medical services such as technicians, porters and laboratory clinicians. This may therefore also be an independent aspect for the hospital to consider when developing service design to improve perinatal outcomes. Whilst continuously performing emergency cesarean section has significant demands on resource utilisation and may be a non-modifiable limiting factor, it is rational to believe that clinical delays to treatment are modifiable even within resource constraints. A previous study, for example, demonstrated that the average time to complete obstetric triage reduced from 192 to $38 \mathrm{~min}$ when a midwife was allocated to this specific task [22].

The high volume of deliveries and baseline incidence of adverse events at Mulago Hospital meant that, although temporally short, our study was sufficiently powered for the crucial outcomes of perinatal and neonatal mortality. The high baseline incidence of adverse outcomes in our study ( $\sim 5 \%$ maternal adverse outcomes and $\sim 10 \%$ perinatal mortality) are in keeping with previously reported outcomes from the study centre $[25,26]$. Furthermore, since such a high volume of deliveries can complicate detailed medical

Table 4 Outcomes of delivery

\begin{tabular}{|c|c|c|c|c|}
\hline Delivery outcome & Number (\%) & $\begin{array}{l}\text { Influence of decision- } \\
\text { to-delivery interval }\end{array}$ & $\begin{array}{l}\text { Influence of } \\
\text { decision time }\end{array}$ & $\begin{array}{l}\text { Influence of } \\
\text { delivery time }\end{array}$ \\
\hline Adverse maternal outcome & $16(4.7 \%)$ & $p=0.26$ & $p=0.61$ & $p=0.54$ \\
\hline Fresh stillbirth & $13(3.8 \%)$ & $p=0.15$ & $p=0.16$ & $p=0.07$ \\
\hline Neonatal death & $21(6.4 \%)$ & $p=0.85$ & $p<0.05^{*}$ & $p=0.07$ \\
\hline Perinatal death & $35(10.2 \%)$ & $p=0.68$ & $p<0.01^{* *}$ & $p<0.05^{*}$ \\
\hline Admission to the Special Care Baby Unit & $78(23.6 \%)$ & $p=0.75$ & $p=0.59$ & $p=0.53$ \\
\hline $\mathrm{APGAR}<7$ at $1 \mathrm{~min}$ & $76(23.0 \%)$ & $p=0.57$ & $p=0.16$ & $p=0.47$ \\
\hline APGAR $<7$ at 5 mins & $77(23.3 \%)$ & $p=0.56$ & $p=0.13$ & $p=0.39$ \\
\hline Other adverse neonatal outcome & $47(14.2 \%)$ & $p=0.24$ & $p<0.05^{*}$ & $p<0.05^{*}$ \\
\hline
\end{tabular}

The number of mothers who experienced each adverse outcome is shown along with the percentage. For outcomes that can apply to all deliveries (perinatal death, stillbirth, adverse maternal outcomes) the total was $n=344$. For outcomes that apply only to live born infants, the total was $n=330$. More than one adverse outcome was present in some cases. The influence of decision-to-delivery interval is represented by $p$-values derived from logistic regression models, in which the risk of outcome is conditioned upon the length of the interval in hours. The influence of decision time and delivery time are the $p$-values derived from generalised additive models with a non-parametric effect for the time of day at decision and delivery respectively. All models were adjusted for the sex and gestational age-specific centile of the neonate's birth weight. ${ }^{*} p<0.05,{ }^{* *} p<0.01$ 
A

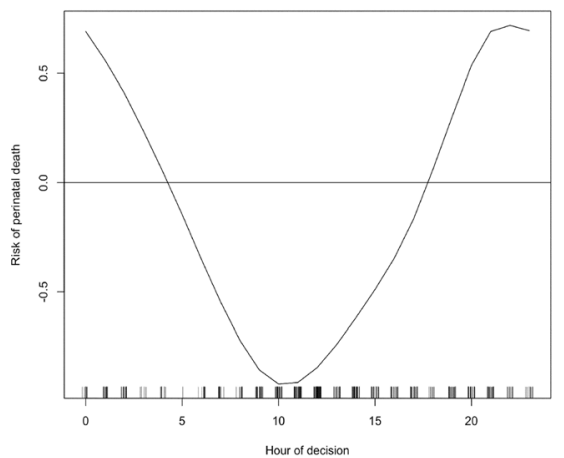

C

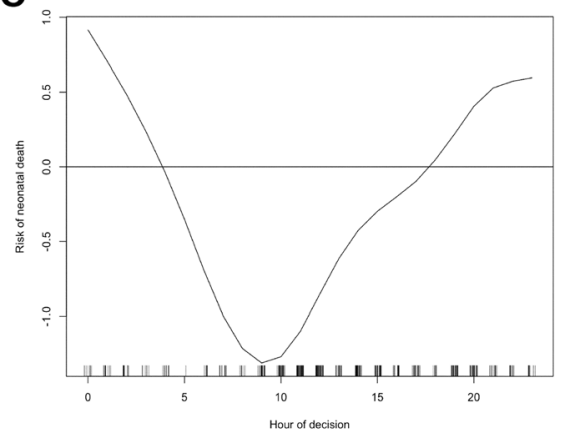

B

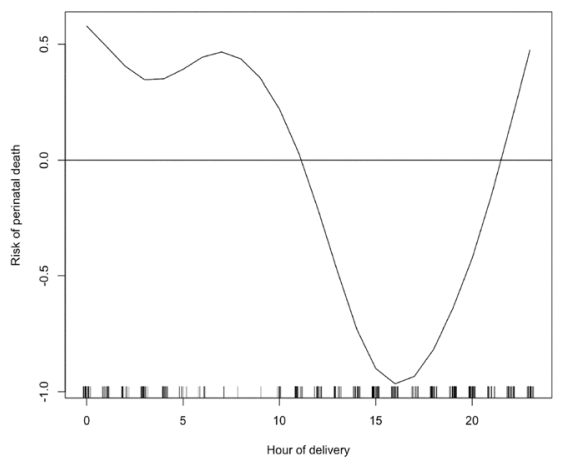

D

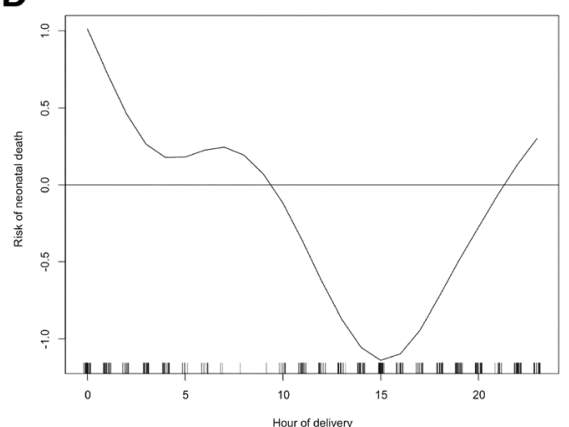

Fig. 5 Risk of adverse perinatal outcomes by time of day. a Risk of perinatal death by hour of decision making. The risk of perinatal death was significantly higher than average for neonates where the decision to deliver by emergency cesarean section was made at night (20:00-02:00) and significantly lower than average where the decision was made in the morning $(08: 00-12: 00), p<0.01$. b Risk of perinatal death by hour of delivery. The risk of perinatal death was significantly higher than average for neonates delivered at night (24:00-08:00) and significantly lower than average where delivery was in the afternoon (14:00-18:00), $p<0.05$. c) Risk of neonatal death by hour of decision making. The risk of neonatal death was significantly higher than average for neonates where the decision to deliver by emergency cesarean section was made at night (24:00-02:00) and significantly lower than average where the decision was made in the morning (06:00-12:00), $p<0.05$. d) Risk of neonatal death by hour of delivery. The risk of neonatal death was not significantly higher than average for neonates delivered at night, however, was significantly lower than average where delivery was in the afternoon (13:00-17:00), $p<0.05$

record-keeping, our strategy of bespoke contemporaneous data collection by a dedicated researcher present in the institution increases our confidence in the accuracy of the timings presented. The study is also underpinned by a powerful and sophisticated statistical modelling strategy, in which non-parametric dynamic additive models were used to determine the risks of adverse perinatal outcomes relative to baseline risk, without making assumptions about the risk / time relationship.

A limitation of the current study is that our results relate only to delay in delivery after the decision for emergency cesarean is made. Previous analysis from the study setting has found that the average triage time is longer for mothers who present overnight [22]. Delays besides the decision-to-delivery interval may therefore vary in a predictable diurnal cycle. We were also unable to explicitly model the influence of the experience of available obstetricians throughout the day. Whilst the periods during which the least experienced obstetricians are alone in the hospital correlate with the longest decision- to-delivery intervals and highest risk of perinatal mortality, we could not demonstrate a causal association here. A further limitation is the absence of a routinely applied categorisation of urgency for non-elective caesareans in the study context. Adoption of such a system could help to identify and prioritise higher risk cases, providing further scope for reducing perinatal mortality. While our study utilises a large cohort, there was insufficient power to analysis the relationship between decision-to-delivery interval and perinatal outcome separately for each indication for caesarean section. It might be expected that for some indications, particularly those in which delivery is very urgent such as cord prolapse, that a more direct relationship might exist. For other indications, such as fetal malpresentation, the findings of sub-group analyses would be likely consistent with the full cohort data.

In this context, where many mothers present to hospital already in obstructed labour [27], a direction of future study would be to investigate delays in the total time to delivery besides the decision-to-delivery interval. 
Such delays may also vary diurnally, for example, due to traffic patterns in the surrounding urban area. Clinician experience can be associated with perinatal outcomes both indirectly, through increased decision-to-delivery interval and directly, through for example operative skill. Further analysis of the obstetricians available throughout the day is required to determine whether modifying the periods during which the those least experienced are alone in the hospital would result in reduced risk of perinatal mortality. Future analyses in this area should also include analysis of factors potentially contributing to adverse outcomes after the decision for emergency caesarean section has been made.

\section{Conclusion}

In this busy sub-Saharan Africa maternity setting, the average decision-to-delivery interval of emergency cesarean section is longer than target times adopted in well-resourced obstetric settings. There is no direct relationship between the interval and adverse perinatal outcomes, however, there is significant diurnal variation in the risk of perinatal and neonatal mortality. The rate of adverse perinatal outcomes is better correlated with time of decision-making than with length of decision-to-delivery interval or time of delivery. This suggests that focus on supporting safe clinical decision-making during high-risk periods may be a useful and feasible strategy for reducing neonatal morbidity and mortality.

\section{Abbreviations}

HR: Hazard ratio; OR: Odds ratio; Cl: Confidence interval

\section{Acknowledgements}

Not applicable.

\section{Authors' contributions}

The project was conceived by $\mathrm{NH}$ and $\mathrm{CA}$, with guidance from AN and AM. The research protocol for permission to use medical records was written by $\mathrm{NH}$ and IN and ethical approval was obtained by IN and MS from the Research and Ethics Committee of Mulago Hospital. Data was collected by $\mathrm{NH}$, facilitated and supervised by MS at Mulago Hospital. Data analysis was conducted by $\mathrm{CA}$ and the manuscript was written by $\mathrm{NH}$ and $\mathrm{CA}$. Intellectual input and approval of the manuscript was provided by IN, AN, MS, AM and CP. All authors have read and approved the manuscript.

\section{Funding}

CA is supported by an Isaac Newton Trust [12.21(a)]/Wellcome Trust ISSF [105602/Z/14/Z]/ University of Cambridge Joint Research Grant. This work was supported by NURTURE fellowship to AN, grant number D43TW010132 and the DELTAS Africa Initiative (grant\# 107743/Z/15/Z). The DELTAS Africa Initiative is an independent funding scheme of the African Academy of Sciences (AAS)'s Alliance for Accelerating Excellence in Science in Africa (AESA) and supported by the New Partnership for Africa's Development Planning and Coordinating Agency (NEPAD Agency) with funding from the Wellcome Trust (grant \#107743/Z/15/Z) and the UK government. The views expressed in this publication are those of the author(s) and not necessarily those of AAS, NEPAD Agency, Wellcome Trust or the UK government. NH was supported by a financial contribution from the Addenbrooke's Abroad Grant Scheme, provided by Addenbrooke's Hospital Charitable Trust. The funders had no role in the design or conduct of this study.

\section{Availability of data and materials}

The datasets generated and analysed during the current study are not publicly available due to the terms of the ethical approval under which they were obtained, but may be available from the corresponding author on reasonable request.

\section{Ethics approval and consent to participate}

Ethical approval for the study was granted by the Mulago Hospital Research and Ethics Committee (protocol number 1605). A waiver for the requirement of informed consent from the mothers whose records were analysed was granted by the Chair of the Committee on the grounds of being a minimal risk study.

Consent for publication

Not applicable.

\section{Competing interests}

The authors have no conflicts of interest to declare.

\section{Author details}

${ }^{1}$ School of Clinical Medicine, University of Cambridge, NIHR Cambridge Comprehensive Biomedical Research Centre, Cambridge CB2 OSW, UK. ${ }^{2}$ Department of Obstetrics and Gynecology, Makerere University and Mulago National Referral Hospital, Kampala, Uganda. ${ }^{3}$ Department of Pathology and Centre for Trophoblast Research, University of Cambridge, Cambridge CB2 3EG, UK. ${ }^{4}$ Department of Obstetrics and Gynecology, Box 223, The Rosie Hospital, Cambridge CB2 OSW, UK. ${ }^{5}$ University Department of Obstetrics and Gynecology, University of Cambridge, NIHR Cambridge Comprehensive Biomedical Research Centre, Cambridge CB2 OSW, UK.

Received: 27 August 2019 Accepted: 11 May 2020 Published online: 27 May 2020

\section{References}

1. Lee KS, Park SC, Khoshnood B, Hsieh HL, Mittendorf R. Human development index as a predictor of infant and maternal mortality rates. J Pediatr. 1997: 131(3):430-3.

2. WHO. World Health Organization ICD-10: International Statistical Classification of Diseases and Related Health Problems, Tenth Revision Geneva: World Health Organization; 1992Available from: www.who.int/ classifications/icd/Accessed 24 June 2013.

3. Alkema L, Chou D, Hogan D, et al. Global, regional, and national levels and trends in maternal mortality between 1990 and 2015, with scenario-based projections to 2030: a systematic analysis by the UN maternal mortality estimation inter-agency group. Lancet. 2016;387(10017):462-74.

4. Akseer N, Lawn JE, Keenan W, et al. Ending preventable newborn deaths in a generation. Int J Gynaecol Obstet. 2015;131(Suppl 1):S43-8.

5. Koblinsky M, Moyer CA, Calvert C, et al. Quality maternity care for every woman, everywhere: a call to action. Lancet. 2016;388(10057):2307-20.

6. Aiken CE, Aiken AR, Scott JG, Brockelsby JC. The influence of hours worked prior to delivery on maternal and neonatal outcomes: a retrospective cohort study. Am J Obstet Gynecol. 2016;215(5):634 e631-7.

7. Reif $P$, Pichler $G$, Griesbacher $A$, et al. Do time of birth, unit volume, and staff seniority affect neonatal outcome in deliveries at $>/=34(+0)$ weeks of gestation? BJOG. 2018;125(7):884-91.

8. Tolcher MC, Johnson RL, El-Nashar SA, West CP. Decision-to-incision time and neonatal outcomes: a systematic review and meta-analysis. Obstet Gynecol. 2014;123(3):536-48.

9. Royal College of Obstetricians and Gynaecologists, UK. Good Practice No. 11 Classification of urgency of Caesarean section - a continuum of risk April 2010

10. American Academy of Pediatrics, American College of Obstetricians and Gynecologists. Guidelines for perinatal care. 8th ed. Elk Grove Village (IL): AAP. Washington, DC: American College of Obstetricians and Gynecologists; 2017.

11. Boehm FH. Decision to incision: time to reconsider. Am J Obstet Gynecol. 2012;206(2):97-8.

12. MacKenzie IZ, Cooke I. What is a reasonable time from decision-todelivery by caesarean section? Evidence from 415 deliveries. BJOG. 2002;109(5):498-504. 
13. Singh $R$, Deo S, Pradeep Y. The decision-to-delivery interval in emergency caesarean sections and its correlation with perinatal outcome: evidence from 204 deliveries in a developing country. Trop Dr. 2012;42(2):67-9.

14. Nolens B, Namiiro F, Lule J, van den Akker T, van Roosmalen J, Byamugisha J. Prospective cohort study comparing outcomes between vacuum extraction and second-stage cesarean delivery at a Ugandan tertiary referral hospital. Int J Gynaecol Obstet. 2018;142(1):28-36.

15. Ssempiira J, Nambuusi B, Kissa J, et al. Geostatistical modelling of malaria indicator survey data to assess the effects of interventions on the geographical distribution of malaria prevalence in children less than 5 years in Uganda. PLoS One. 2017;12(4):e0174948.

16. Nakimuli A, Nakubulwa $S$, Kakaire $O$, et al. Incidence and determinants of neonatal morbidity after elective caesarean section at the national referral hospital in Kampala, Uganda. BMC Res Notes. 2015;8:624.

17. Beaton A, Okello E, Scheel A, DeWyer A, Ssembatya R, Baaka O, Namisanvu H, Njeri A, Matovu A, Namagembe I, Mccarter R, Carapetis J, DeStigter K, Sable C. Impact of heart disease on maternal, fetal and neonatal outcomes in a low-resource setting. Heart. 2019;105(10):755-60. https://doi.org/10. 1136/heartjil-2018-313810. Epub 2018 Nov 10.

18. Wanyama R, Obai G, Odongo P, Kagawa MN, Baingana RK. Are women in Uganda gaining adequate gestational weight? A prospective study in low income urban Kampala. Reprod Health. 2018;15(1):160.

19. Kisuule I, Kaye DK, Najjuka F, et al. Timing and reasons for coming late for the first antenatal care visit by pregnant women at Mulago hospital, Kampala Uganda. BMC Pregnancy Childbirth. 2013;13:121.

20. Roberts DA, Ng M, Ikilezi G, et al. Benchmarking health system performance across regions in Uganda: a systematic analysis of levels and trends in key maternal and child health interventions, 1990-2011. BMC Med. 2015;13:285.

21. Abdallah Y, Namiiro F, Mugalu J, Nankunda J, Vaucher Y, McMillan D. Is facility based neonatal care in low resource setting keeping pace? A glance at Uganda's National Referral Hospital. Afr Health Sci. 2016;16(2):347-55.

22. Forshaw J, Raybould S, Lewis E, et al. Exploring the third delay: an audit evaluating obstetric triage at Mulago National Referral Hospital. BMC Pregnancy Childbirth. 2016;16(1):300.

23. Hirani BA, McHome BL, Mazuguni NS, Mahande MJ. The decision delivery interval in emergency caesarean section and its associated maternal and fetal outcomes at a referral hospital in northern Tanzania: a cross-sectional study. BMC Pregnancy Childbirth. 2017;17(1):411.

24. Schauberger CW, Chauhan SP. Emergency cesarean section and the 30minute rule: definitions. Am J Perinatol. 2009;26(3):221-6.

25. Musooko M, Kakaire O, Nakimuli A, et al. Incidence and risk factors for early neonatal mortality in newborns with severe perinatal morbidity in Uganda. Int J Gynaecol Obstet. 2014;127(2):201-5.

26. Nakimuli A, Mbalinda SN, Nabirye RC, et al. Still births, neonatal deaths and neonatal near miss cases attributable to severe obstetric complications: a prospective cohort study in two referral hospitals in Uganda. BMC Pediatr. 2015;15:44.

27. Kayiga H, Ajeani J, Kiondo P, Kaye DK. Improving the quality of obstetric care for women with obstructed labour in the national referral hospital in Uganda: lessons learnt from criteria based audit. BMC Pregnancy Childbirth. 2016;16(1):152

\section{Publisher's Note}

Springer Nature remains neutral with regard to jurisdictional claims in published maps and institutional affiliations.

\section{Ready to submit your research? Choose BMC and benefit from:}

- fast, convenient online submission

- thorough peer review by experienced researchers in your field

- rapid publication on acceptance

- support for research data, including large and complex data types

- gold Open Access which fosters wider collaboration and increased citations

- maximum visibility for your research: over $100 \mathrm{M}$ website views per year

At $\mathrm{BMC}$, research is always in progress.

Learn more biomedcentral.com/submissions 